Article

\title{
The Feasibility of Attention Training for Reducing Mind-Wandering and Digital Multitasking in High Schools
}

\author{
Alissa J. Mrazek*(D), Michael D. Mrazek*(D), Peter C. Carr, Alex M. Delegard, Margaret G. Ding, \\ Daniel I. Garcia, Jenna E. Greenstein, Arianna C. Kirk, Erika E. Kodama, Miel J. Krauss, \\ Alex P. Landry ${ }^{\mathbb{D}}$, Crystal A. Stokes, Kyla D. Wickens, Kyle Wong and Jonathan W. Schooler \\ Department of Psychological \& Brain Sciences, University of California, Santa Barbara, \\ Santa Barbara, CA 93106, USA; petecarr@ucsb.edu (P.C.C.); adelegard@gmail.com (A.M.D.); \\ m_ding@ucsb.edu (M.G.D.); danielg@ucsb.edu (D.I.G.); jgreenstein@ucsb.edu (J.E.G.); \\ ariannackirk@gmail.com (A.C.K.); ekodama@ucsb.edu (E.E.K.); mjkrauss@ucsb.edu (M.J.K.); \\ landry.alex1@gmail.com (A.P.L.); castokes@ucsb.edu (C.A.S.); kyla.wickens@emory.edu (K.D.W.); \\ kyle_wong@ucsb.edu (K.W.); jonathanwschooler@gmail.com (J.W.S.) \\ * Correspondence: alissa.mrazek@psych.ucsb.edu (A.J.M.); mrazek@ucsb.edu (M.D.M.)
}

Received: 16 July 2020; Accepted: 5 August 2020; Published: 6 August 2020

\begin{abstract}
During academic activities, adolescents must manage both the internal distraction of mind-wandering and the external distraction of digital media. Attention training has emerged as a promising strategy for minimizing these distractions, but scalable interventions that can deliver effective attention training in high schools are still needed. The present investigation used a one-group pre-post design to examine the feasibility and outcomes of a digital attention training course at a public high school. The intervention was delivered with reasonably strong fidelity of implementation, with students completing $92 \%$ of the lessons and $79 \%$ of the daily exercises. At baseline, students reported mind-wandering more frequently during class than they multitasked, and mind-wandering was more negatively correlated with classroom focus. From pre-test to post-test $(n=229)$, students reported improved emotional regulation and reduced mind-wandering during daily life. Among the $76 \%$ of students who felt they paid attention in class less than they should, classroom focus improved significantly. During class, these students reported significantly less mind-wandering but slightly greater digital multitasking. During homework, they reported significantly less digital multitasking but only marginally reduced mind-wandering. Collectively, these results suggest that online interventions could be a scalable way of providing attention training in high schools, but that future work must consider the role of both mind-wandering and digital multitasking.
\end{abstract}

Keywords: attention; mind-wandering; multitasking; focus; emotion regulation; high school

\section{Introduction}

Attention is an essential basis of learning. To focus during academic activities, students must filter through a tremendous amount of distracting information coming from both the external environment and their own minds. One particularly pervasive source of distraction is mind-wandering, which is known to be highly prevalent in academic settings and disruptive to learning [1,2]. More recently, distraction from digital media has also become a prominent issue [3,4]: $99.9 \%$ of adolescents in the United States use digital media on a daily basis, and nearly half of high school teachers report that digital media significantly distracts students and interferes with learning [4-6]. 
Although restrictive electronic device and internet policies can be useful for limiting digital media distractions, these policies are often difficult to enforce [6,7]. Meanwhile, the disruptive influence of mind-wandering is arguably even harder to limit because it is unobservable to educators [8]. Given the ubiquity of distraction, strategies for helping students train their attention could have important implications for increasing focus and academic achievement.

Considerable research indicates that attention is also relevant to students' mental and emotional health. Influential models of emotion regulation emphasize that individuals can influence their emotional states by choosing where they direct their attention or by using attention to influence their cognitive appraisals [9]. Attentional biases also play an important role in perpetuating mental illness, as when a proclivity to attend to negative information predicts worsening of depression over time [10]. Mental health issues can also be exacerbated by frequently dividing one's attention across multiple media sources, a behavior known as media multitasking [11]. Given rising rates of mental illness among adolescents, methods for helping high school students train their attention have merit not only for enhancing academic achievement but also for promoting mental and emotional health [12].

Attention training can take various forms. Brain training games-perhaps most recognizably provided by the subscription-based service Lumosity-are reportedly used by tens of millions, though evidence for their effectiveness in enhancing attention is minimal [13]. Within clinical settings, attentional bias training shows promise as a way of helping individuals override the tendency to attend to specific stimuli that perpetuate their mental illness [14]. Yet in educational settings, the most widely researched form of training attention is mindfulness-based attention training, which typically involves both the development of attentional skills as well as instruction on how to apply these skills to relate effectively to thoughts and emotions [15]. Although additional research in school settings is needed, a growing body of evidence suggests that this form of attention training can reduce mind-wandering, enhance performance of academic tasks, improve emotional regulation, and promote greater mental health [16-18].

While training attention represents a promising approach for improving the focus and well-being of adolescents, only a small fraction of high school students ever receives this training. Bridging this science-to-service gap will require the development of scalable and evidence-based training programs that can feasibly be integrated into school settings. Digital interventions in particular can circumvent many of the logistical and financial constraints involved in providing effective training to millions of high school students [19]. However, there has been little research evaluating digital attention training programs in high school settings. In one of the only published reports, 1 of 85 students completed the entire intervention (where completion was defined as doing at least 40 of the 96 exercises) [20]. Accordingly, the development and empirical evaluation of attention training programs that are delivered digitally in high school settings is still needed.

The present research entailed two primary goals. The first goal was to further investigate the feasibility and efficacy of Finding Focus, an online course designed to help adolescents train their attention. A recent study found that this course could be delivered with reasonably high fidelity of implementation in a high school setting while highlighting a variety of preliminary positive outcomes, including improved emotional regulation and greater classroom focus among students who reported at baseline that they focused in class less than they should [21]. Although this initial study was promising, replicating findings is a crucially important part of advancing scientific understanding and earning the trust of educators and policy makers [22,23]. The present investigation therefore sought to replicate this prior work.

The second goal was to empirically differentiate the internal distraction of mind-wandering and the external distraction of digital multitasking. Specifically, we examined whether mind-wandering or digital multitasking played a more consequential role in influencing students' classroom focus, and whether the intervention affected these two forms of distraction in similar or different ways. To our knowledge, no research has investigated the influence of these two forms of distraction on high school students' academic focus. We hypothesized that both forms of distraction would be common and 
detrimental to classroom focus. We additionally hypothesized that among the students who reported paying attention in class less than they should, levels of mind-wandering during class would decrease from pre-test to post-test. Because Finding Focus currently includes detailed instruction on managing internal distractions but no explicit instruction on managing external distractions of digital media, we had no specific hypotheses about the effect of the intervention on digital multitasking.

\section{Materials and Methods}

Research design: This replication study used a one-group pre-post design. The research was approved by the Human Subjects Committee at the host university (protocol \# 5-20-0239), and informed consent was obtained from all students and their guardians.

Procedure: Students completed an anonymous online survey before and after the course. All data, with the exception of intervention adherence, were gathered in these surveys. The digital learning platform recorded completion rates for course lessons and exercises, which was used to measure intervention adherence.

Participants: This study was conducted at a public high school in the Northwest region of the United States. The school consists of roughly 600 9-12th grade students. All teachers were notified about the study, and five teachers volunteered to share the course with their students. In total, the course was shared with 309 students in the fall semester. All freshmen completed the course, as well as some students in grades 10-12 enrolled in either AP Physics or a Special Education class.

Teachers who were facilitating the course were encouraged to invite their students to participate in the research surveys. A total of 317 students completed the pre-test. Teachers were repeatedly asked to share the post-test survey with every student who completed the pre-test regardless of course adherence. A total of 235 students completed the post-test. This attrition was largely driven by one teacher who chose not to administer the post-test to her cumulative 65 students. Adherence data from these students were still available and are reported.

All available data were included in analyses. Both pre-test and post-test data were available for 229 students. Demographic information was collected at pre-test. There were 161 freshmen, eight sophomores, 73 juniors, 67 seniors, and five declined to answer. One hundred and ninety-five participants identified as male, 103 identified as female, two identified as non-binary, and 14 preferred not to say. The number of students identifying with a specific race was as follows: Asian-183 students; Caucasian-69; African American/Black-4; American Indian/Alaskan Native—4; Native Hawaiian or Other Pacific Islander-2; mix of two or more races-22; 25 students preferred not to say. Twelve students identified as Hispanic.

Intervention: Students received attention training via an online course called Finding Focus. The course was delivered through a custom digital learning platform that allowed students to access the course on computers, tablets, or phones. The course provided students with repeated practice in the skills of (i) focusing on one specific aspect of their experience while (ii) inhibiting the tendency to become distracted by other thoughts and perceptions. It also provided students with detailed guidance on how to use these skills to have more influence over their thoughts, evaluations, and emotions. There was no explicit instruction regarding multitasking with digital media. The entire course included $2.5 \mathrm{~h}$ of content, including four 12-min lessons and daily 4-min exercises. Content unlocked over 22 days, with one lesson unlocking each week and an exercise unlocking each day. Teachers were encouraged to have students complete the lessons and daily exercises during class.

The course was designed to help students train their attention and learn how to use attention to relate more effectively to thoughts, evaluations, and emotions. The course lessons presented three fundamental skills: anchoring, focusing, and releasing. Anchoring was defined as intentionally deciding where to focus. Focusing was defined as directing your attention to the anchor you chose. Releasing was defined as letting go of a distraction by not giving it any more attention.

These three fundamental skills were trained through daily exercises. The course featured several kinds of daily exercises that each involved focusing on a specific aspect of experience, such as the 
sensations of breathing or the sounds of music. Students were encouraged to deliberately anchor their attention on the relevant aspect of their experience, focus their attention on that experience, and release all distracting thoughts and perceptions. Students also learned how to use these three skills in daily life by applying specific strategies such as re-focusing (releasing a counterproductive thought and choosing a more worthwhile anchor) and re-evaluating (releasing an unhelpful evaluation and focusing on a more empowering one). As such, the intervention had a strong emphasis on training students to reduce the impact of internal distractions.

The entire intervention was delivered using a custom digital learning platform that provided content tailored to the interests of individual users. For example, students indicated their preferred music genre and then received daily exercises in this genre. Each student completed the intervention independently. The digital learning platform provided teachers with an interface to track student progress throughout the course.

Measures: Validated self-report instruments were used whenever possible. In cases where no validated instrument existed to address the specific research question of interest, researcher-developed measures were used. All of these measures were written to maximize face validity using vocabulary that is appropriate for adolescents. The order of instruments was randomized.

Fidelity of implementation (FOI): The intervention's FOI was objectively monitored through the digital learning platform, which automatically records whether and when every student completes each lesson and exercise of the intervention. Only full completion was recorded, and partial completion counted as incomplete. The primary indicator of FOI was the proportion of all lessons and exercises successfully completed by students within the allocated time frame of the study.

Ideal and actual classroom focus: Relatively few students believe that they should always pay attention in class, so two questions were used to capture the distinction between how much a student reports paying attention versus how much they feel they ideally should pay attention [21]. The first question asked, "On average across all your classes, how often do you keep your undivided attention focused on class?" The second question was stated as follows: "This next question is NOT about what other people think you should do. It's about what you believe is best for yourself. On average across all your classes, how often would you ideally keep your undivided attention focused on class?". Both questions were asked on a scale from $0 \%$ of the time to $100 \%$ of the time. Each question was utilized as a discrete measure. Prior research suggests that the discrepancy between these two answers (e.g., how often would you ideally keep your undivided attention focused on class minus how often do you keep your undivided attention focused on class) can serve as a useful way to identify which students see room for improvement [21].

Academic distraction: Four questions assessed students' tendencies to mind-wander or multitask with digital devices during academic activities: (1) “While I' $m$ in class, I mind-wander or daydream about things unrelated to class", (2) "While I'm doing homework, I mind-wander or daydream about things unrelated to my homework", (3) "While I'm in class, I use my electronic devices for things unrelated to class", and (4) "While I'm doing homework, I use my electronic devices for things unrelated to my homework". For all four questions, students responded on a scale of 1-Rarely, 2-Sometimes, 3-Often, and 4-Very Often. Each question was utilized as a discrete measure.

Mindsets about focus: The Mindsets about Focus Scale is a 9-item measure [21]. The instrument evaluates whether an individual (i) believes their ability to focus can improve through training (growth mindset subscale), (ii) is motivated to train their ability to focus (motivation subscale), and (iii) is confident that they know how to train their attention (self-efficacy subscale). The growth mindset subscale consisted of three questions, including "My ability to focus is a skill that can get much better with practice" (pre-test: $a=0.84$; post-test: $a=0.87$ ). The motivation subscale entailed three questions, including "I will do whatever it takes to improve my ability to focus" (pre-test: $a=0.82$; post-test: $a=0.92$ ). The self-efficacy subscale had three items including "I know exactly what to do to increase my ability to focus" (pre-test: $a=0.80$; post-test: $a=0.83$ ). All questions were answered on a 
scale of 1-Strongly Disagree to 6-Strongly Agree, and items within each subscale were averaged into composite scores.

Mind-wandering in daily life: The Mind-Wandering Questionnaire (MWQ) is a 5-item instrument measuring trait levels of mind-wandering on a scale of 1-Almost Never to 6-Almost Always ("I find myself listening with one ear, thinking about something else at the same time"; pre-test: $a=0.77$; post-test: $a=0.86$ ). The MWQ has been validated with both adults and adolescents [17].

Emotion regulation: The Emotion Regulation Questionnaire for Children and Adolescents (ERQ-CA) is a version of the Emotion Regulation Questionnaire that is adapted to be more appropriate for ages 10-18 [24,25]. This scale consists of two subscales assessing cognitive reappraisal ("I control my feelings about things by changing the way I think about them") and expressive suppression ("When I'm feeling bad (e.g., sad, angry, or worried), I'm careful not to show it"). All questions were answered on a scale of 1-Strongly Disagree to 6-Strongly Agree (pre-test: $a=0.87$; post-test: $a=0.92$ ). Given ambiguity regarding the appropriateness of expressive suppression as a healthy strategy for emotion regulation, only the cognitive reappraisal subscale was included.

Life demands: Current demands were assessed by adapting an existing 1-item measure [26]. The item asked, "Over the last 7 days, how much have you had on your plate to deal with (e.g., homework, exams, managing relationships, extracurricular commitments, health challenges, etc.)?" on a five-point scale from 1 (way less than usual) to 5 (way more than usual).

Stress and stress management: As used in previous research [21], two questions were used to capture the distinction between experiencing stress and effectively managing that stress. Participants first responded to the question, "Over the last two weeks, how stressed have you been?" on a scale from 0-100 where higher values indicated more stress. Participants then responded to the question, "Over the last two weeks, how well have you managed your stress?" on a scale from 0-100 where higher values indicated better stress management.

Data analysis overview: The feasibility of the intervention was evaluated as the percentage of course lessons and exercises that students completed. The assessment of student outcomes was done using the remaining quantitative measures. Paired t-tests were used to examine changes in quantitative data from pre-test to post-test. Where appropriate, linear regression was used to assess the relative strength of various predictors when included in a single model.

\section{Results}

\subsection{Goal 1: Replication of the Intervention's Feasibility, FOI, and Preliminary Efficacy}

Fidelity of implementation: Ninety-five per cent of students reported that their teacher set a clear expectation that they should complete the lessons and daily exercises. Seventy-one per cent of students said that they were given some sort of credit for completing the course. Due to time constraints, one teacher chose not to continue facilitating the course part way through sharing it. This resulted in two of the ten classes not completing the course nor the post-test.

The digital learning platform recorded completion of lessons and daily exercises for all students who created accounts regardless of whether they completed the pre-test or post-test. Due to the anonymous nature of the survey data, adherence data could not be linked to survey data. On average, $92 \%$ of lessons were completed ( $97 \%$ for lesson $1 ; 96 \%$ for lesson $2 ; 91 \%$ for lesson 3; $88 \%$ for lesson 4). Students completed $79 \%$ of the daily exercises. Table 1 presents completion rates by group. In some cases, multiple classrooms were facilitated as a single group within the digital learning platform, preventing breakdown of adherence data by classroom.

Mindsets about focus: Replicating previous work [21], the course led students to more strongly endorse a growth mindset regarding their ability to focus, $t(228)=4.01, p<0.001, d=0.28$ (Figure 1). Students also became more confident that they knew how to train this ability, $t(228)=9.97, p<0.001$, $d=0.83$. However, there was no change in students' motivation to train their ability to focus, $t(228)=1.33, p=0.19, d=0.08$. 
Table 1. Adherence rates in lessons and daily exercises.

\begin{tabular}{lccc}
\hline \multicolumn{1}{c}{ Group } & $\boldsymbol{n}$ & Lesson Completion & Exercise Completion \\
\hline AP Physics (2 classes) & 65 & $100 \%$ & $99.6 \%$ \\
Special Education (1 class) & 15 & $98.3 \%$ & $98.4 \%$ \\
Freshman (5 classes) & 165 & $98.4 \%$ & $92.4 \%$ \\
Environmental Science (2 classes) & 65 & $70 \%$ & $25 \%$ \\
\hline
\end{tabular}

Note: Completion rates of lessons and exercises across all students in a given group. Groups are listed by descending levels of adherence. The Environmental Science classes did not finish the course or complete the post-test due to time constraints. $n$ refers to the sample size of each group.

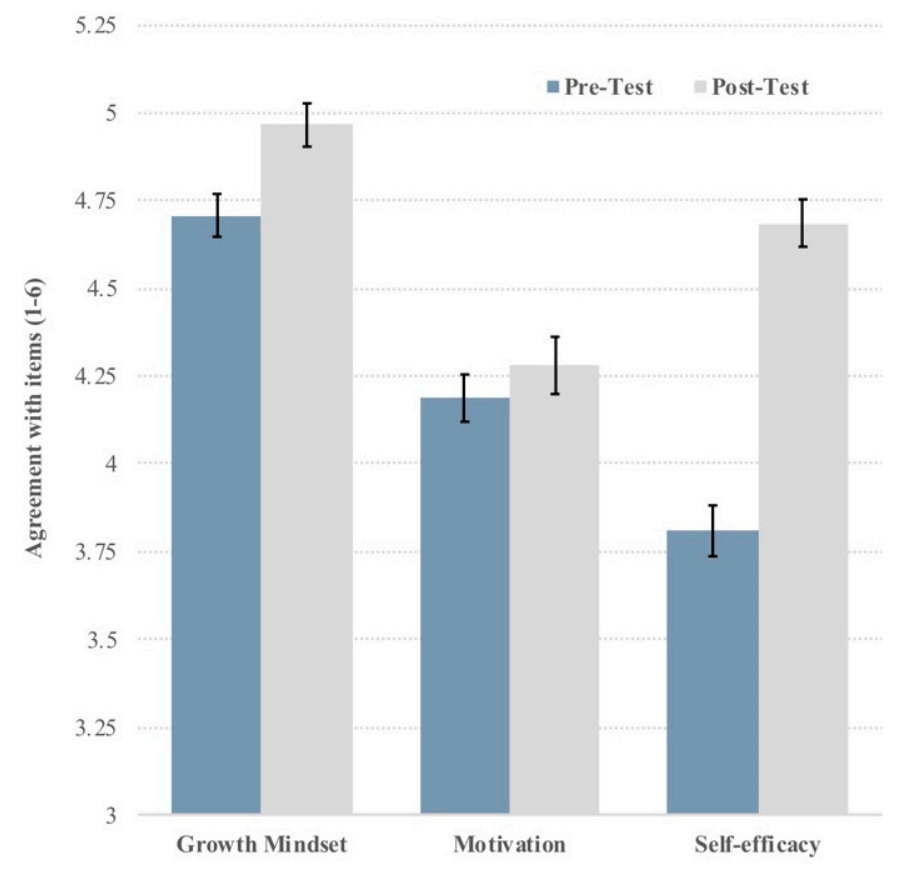

Figure 1. Changes in mindsets about focus from pre-test to post-test. Changes in mindsets about one's ability to focus. At pre-test and post-test, students indicated (1) whether they believed their ability to focus could improve through training, (2) how motivated they were to train their ability to focus, and (3) how confident they were in knowing how to train their focus. Error bars represent plus or minus one standard error.

Mind-wandering during daily life and ability to focus: Student levels of mind-wandering during daily life significantly decreased from pre-test $(\mathrm{M}=3.49, \mathrm{SD}=0.91)$ to post-test $(\mathrm{M}=3.32, \mathrm{SD}=1.00)$, $t(177)=2.97, p<0.01, d=0.17$. When asked whether or not the course improved their ability to focus, $73 \%$ of students reported that the course enhanced their focus.

Focus during class: Following Mrazek and colleagues (2019), we predicted that the students most likely to increase their focus during class were the ones who reported a discrepancy at pre-test in how much they paid attention relative to how much they felt they should pay attention. At pre-test, students felt they should ideally keep their undivided attention focused on class $82.88 \%$ of the time $(\mathrm{SD}=17.79)$. They estimated that they actually kept their attention focused on class $69.59 \%$ of the time $(S D=21.15)$. Approximately $76 \%$ of students reported focusing less often than they felt they should.

Among the $76 \%$ of students who reported focusing less often than they should at pre-test, results suggest that actual focus improved significantly from pre-test $(\mathrm{M}=69.00, \mathrm{SD}=19.53)$ to post-test $(\mathrm{M}=72.42, \mathrm{SD}=20.57), t(177)=2.61, p=0.01, d=0.17$. The course did not affect how much students felt they should pay attention during class, $t(228)=0.85, p=0.40$. Across the entire sample, levels of focus did not significantly change, $t(228)=0.90, p=0.37$. 
Emotion regulation: Replicating previous work [21], students showed increased levels of emotion regulation from pre- to post-test (Table 2).

Table 2. Changes in life demands, stress, and emotional regulation.

\begin{tabular}{lccccc}
\hline & Pre-Test M (SD) & Post-Test M (SD) & $\boldsymbol{t}$ & $\boldsymbol{p}$ & Cohen's $\boldsymbol{d}$ \\
\hline Emotion regulation & $3.83(0.98)$ & $4.28(1.09)$ & 7.19 & 0.000 & 0.44 \\
Demands & $4.00(0.85)$ & $3.74(1.00)$ & 3.60 & 0.000 & 0.28 \\
Stress & $61.66(23.82)$ & $56.99(26.63)$ & 2.82 & 0.005 & 0.19 \\
Stress management & $56.89(25.13)$ & $58.90(26.60)$ & 1.12 & 0.26 & 0.08 \\
\hline
\end{tabular}

Note: Paired sample $t$-tests were used to compare pre-test and post-test scores.

Demands and stress: Students experienced significantly decreased levels of stress and demands from pre-test to post-test (Table 2). Changes in stress over time were positively correlated with changes in demands $(r=0.39, p<0.001)$.

Stress management: Across the entire sample, students did not report an increase in how effectively they managed their stress from pre- to post-test (Table 2). However, an exploratory analysis revealed that among the students who experienced greater than average levels of stress at pre-test, the course led to improved stress management from pre-test $(M=48.43, S D=23.96)$ to post-test $(M=55.70$, $\mathrm{SD}=26.16), t(124)=3.10, p=0.002, d=0.29$.

\subsection{Goal 2: Investigation of Mind-Wandering and Digital Multitasking}

Baseline prevalence of mind-wandering and digital multitasking: During class, students' tendencies to mind-wander $(\mathrm{M}=2.36, \mathrm{SD}=0.89)$ were more prevalent than their tendencies to multitask with digital media $(\mathrm{M}=1.50, \mathrm{SD}=0.84)$. However, during homework, students reported similar levels of mind-wandering $(\mathrm{M}=2.48, \mathrm{SD}=0.97)$ and multitasking with digital media $(\mathrm{M}=2.44$, $\mathrm{SD}=1.01)$.

Baseline predictors of classroom focus: At baseline, classroom focus was significantly correlated with both mind-wandering in class $(r=-0.49, p<0.001)$ and digital multitasking in class $(r=-0.25$, $p<0.001)$. However, a multiple regression model predicting classroom focus revealed that only mind-wandering in class remained a significant predictor, $B=-10.94, t(321)=-8.71, p<0.001$. In contrast, multitasking in class only marginally predicted classroom focus, $B=-2.38, t(321)=-1.76$, $p=0.079$. The $95 \%$ confidence intervals for the standardized betas did not overlap, indicating that mind-wandering, $\beta=-0.46,95 \%$ CI $[-0.56,-0.36]$, was a significantly stronger predictor than multitasking, $\beta=-0.09,95 \%$ CI $[-0.20,0.01]$.

Changes in mind-wandering and multitasking during class: As hypothesized, among the students who reported a discrepancy at pre-test in how much they paid attention relative to how much they felt they should pay attention, levels of mind-wandering during class decreased significantly from pre-test $(\mathrm{M}=2.31, \mathrm{SD}=0.87)$ to post-test $(\mathrm{M}=2.18, \mathrm{SD}=0.72), t(176)=2.17, p=0.03, d=0.16$. Contrary to hypotheses, among this subset of students, levels of multitasking during class increased significantly from pre-test $(\mathrm{M}=1.36, \mathrm{SD}=0.72)$ to post-test $(\mathrm{M}=1.47, \mathrm{SD}=0.76), t(176)=-2.11, p=0.04, d=0.15$.

Across the entire sample, students reported a similar level of digital multitasking during class from pre-test to post-test, $t(227)=-1.52, p=0.13$. There was also no change when looking at the entire sample in students' levels of mind-wandering during class from pre-test to post-test, $t(227)=1.56$, $p=0.12$.

Changes in mind-wandering and multitasking during homework. Students reported a significant decrease in their digital multitasking during homework from pre-test $(\mathrm{M}=2.33, \mathrm{SD}=0.99)$ to post-test $(\mathrm{M}=2.21, \mathrm{SD}=0.96), t(227)=1.99, p=0.05, d=0.12$. Students reported a marginal decrease in their mind-wandering during homework from pre-test $(\mathrm{M}=2.42, \mathrm{SD}=0.98)$ to post-test $(\mathrm{M}=2.30$, $\mathrm{SD}=0.91), t(227)=1.81, p=0.07, d=0.13$. 


\section{Discussion}

How well students regulate their attention has important implications for their academic achievement and mental health. Although existing research indicates that attention training can lead to positive outcomes in high school settings, research has yet to establish the feasibility and effectiveness of attention training interventions that are delivered digitally. The present research found that Finding Focus, a 22-day attention training intervention, could be delivered digitally with strong fidelity of implementation in the majority of classrooms. Although the one-group pre-post design precludes definitive conclusions about whether the intervention benefited students, several positive outcomes were observed, including reduced mind-wandering, greater classroom focus, and improved emotional regulation.

One goal of this research was to empirically differentiate between the internal distraction of mind-wandering and the external distraction of multitasking with digital media. During class, students reported mind-wandering more frequently than they multitasked. Furthermore, mind-wandering was the stronger predictor of self-reported classroom focus. One plausible explanation of this finding is that unlike digital media multitasking - which is both observable and to some degree manageable through the electronic device policy enforced by a school or parent-mind-wandering is an inherently internal experience that eludes third-party observation or control [27]. Consistent with this interpretation, in college classrooms that have no restrictions on electronic device use, multitasking can be an even greater distraction than mind-wandering [2].

As predicted, the intervention led to reduced mind-wandering during class among those students who initially reported paying attention in class less than they should. We also observed a non-significant reduction of mind-wandering during homework. The impact of the intervention on multitasking was less consistent. Whereas students reported a reduction in multitasking during homework, they reported an increase in multitasking during class. Although it is unclear what explains this unpredicted increase, one possibility is that the increased multitasking in class was directly related to the significant decrease in mind-wandering. Prior work has found that students are more susceptible to mind-wandering when device use is restricted [28]. If the inverse were also true, then a significant reduction in mind-wandering might increase students' susceptibility to multitasking. This implies that it may be important for interventions to explicitly address both mind-wandering and digital multitasking simultaneously. The intervention evaluated in this investigation had a strong emphasis on helping students learn to deal with the internal distractions of thoughts and emotions, and it may have been more effective if it also addressed the topic of multitasking with digital media.

Attention plays an important role in the management of stress and emotions. Attention not only dictates what aspects of a situation are noticed but also how a situation is appraised [9]. Consistent with this important role of attention in emotion-and replicating the prior feasibility study-we observed that the intervention elicited a significant improvement in emotional regulation. Yet whereas the prior study found marginally increased stress and improved stress management, the present study observed decreased stress and no significant change in stress management. This is likely due to changes in life demands from pre-test to post-test. Whereas in the prior study students experienced increased demands and stress over time, students in the present study experienced decreased demands and stress over time. This suggests that changes in stress management may be confounded with changes in stress and demands, and that future research must take these relationships into account.

Despite the promising feasibility of this intervention in high school settings, this study has several limitations. First, due to the lack of a control group, changes in student outcomes must be considered preliminary. Without a control condition, it cannot be determined whether the intervention or a variety of other confounds, such as student maturation, teacher influence, or testing effects, were responsible for the changes observed. Future work should evaluate the efficacy of Finding Focus using a randomized controlled trial to rule out these alternative explanations. A second limitation is that there was significant attrition from pre-test to post-test. Attrition is most problematic if there is systematic dropout based on individual differences [29]. Given that the majority of attrition was due 
to one specific teacher deciding to not share the post-test survey, the risk of systematic dropout based on individual differences is considerably lower. A third limitation of the present research is the use of several researcher-generated survey items instead of validated self-report instruments. This was required to address the research questions of interest, and these measures were written to maximize face validity using vocabulary that is appropriate for adolescents. Nevertheless, findings from these researcher-generated items should be interpreted as less definitive. Finally, additional studies with larger samples drawn from diverse schools will be necessary to determine to what extent these findings generalize across other student populations.

Given that distraction and mental health issues are on the rise among adolescents, it is imperative to find solutions that can equip teenagers with the skills they need to overcome these challenges $[4,12]$. Attention training has emerged as a promising tool for helping students focus and regulate their emotions, yet making this training widely available entails considerable financial and logistical challenges. Although additional research is needed, this investigation suggests that digital attention training may be a feasible, effective, and scalable way of making attention training widely available to high school students.

Author Contributions: All authors contributed to the design and creation of the attention training intervention and the conceptualization of the study. A.J.M., M.D.M. and J.E.G. contributed to data collection. A.J.M., M.D.M. and A.P.L. contributed to data analysis. P.C.C., K.W. and A.M.D. contributed to the software development. A.C.K., M.J.K. and E.E.K., contributed to the visual design of the intervention. A.J.M., M.D.M., M.G.D., D.I.G., J.E.G., M.J.K., A.P.L., C.A.S. and K.D.W. contributed to the initial drafting of the manuscript. All authors contributed to revising the manuscript. All authors have read and agreed to the published version of the manuscript.

Funding: This research was funded by the U.S. Department of Education's Institute of Education Sciences (grant number R305A170445). The content does not reflect the position or policy of the U.S. government.

Conflicts of Interest: The authors declare no conflict of interest.

\section{References}

1. Smallwood, J.; Fishman, D.J.; Schooler, J.W. Counting the Cost of an Absent Mind: Mind Wandering as an Underrecognized Influence on Educational Performance. Psychon. Bull. Rev. 2007, 14, 230-236. [CrossRef] [PubMed]

2. Wammes, J.D.; Seli, P.; Cheyne, J.A.; Boucher, P.O.; Smilek, D. Mind Wandering during Lectures II: Relation to Academic Performance. Sch. Teach. Learn. Psychol. 2016, 2, 33-48. [CrossRef]

3. Aagaard, J. Media Multitasking, Attention, and Distraction: A Critical Discussion. Phenom. Cogn. Sci. 2015, 14, 885-896. [CrossRef]

4. Rosen, L.D. The Distracted Student Mind-Enhancing Its Focus and Attention. Phi. Delta. Kappan. 2017, 99, 8-14. [CrossRef]

5. Przybylski, A.K.; Weinstein, N. A Large-Scale Test of the Goldilocks Hypothesis: Quantifying the Relations between Digital-Screen Use and the Mental Well-Being of Adolescents. Psychol. Sci. 2017, 28, 204-215. [CrossRef]

6. Vega, V.; Robb, M.B. The Common Sense Census: Inside the 21st-Century Classroom; Common Sense Media: San Francisco, CA, USA, 2019.

7. Beland, L.-P.; Murphy, R.J. Ill Communication: Mobile Phones \& Student Performance; International Workshop on Applied Economics of Education: Catanzaro, Italy, 2014.

8. Smallwood, J.; Schooler, J.W. The Science of Mind Wandering: Empirically Navigating the Stream of Consciousness. Annu. Rev. Psychol. 2015, 66, 487-518. [CrossRef] [PubMed]

9. McRae, K.; Gross, J.J. Emotion Regulation. Emotion 2020, 20, 1-9. [CrossRef]

10. Disner, S.G.; Shumake, J.D.; Beevers, C.G. Self-Referential Schemas and Attentional Bias Predict Severity and Naturalistic Course of Depression Symptoms. Cogn. Emot. 2017, 31, 632-644. [CrossRef]

11. Cheever, N.A.; Peviani, K.; Rosen, L.D. Media Multitasking and Mental Health. In Technology and Adolescent Mental Health; Moreno, M.A., Radovic, A., Eds.; Springer International Publishing: Cham, Switzerland, 2018; pp. 101-112. [CrossRef] 
12. Twenge, J.M. More Time on Technology, Less Happiness? Associations between Digital-Media Use and Psychological Well-Being. Curr. Dir. Psychol. Sci. 2019, 0963721419838244. [CrossRef]

13. Bainbridge, K.; Mayer, R.E. Shining the Light of Research on Lumosity. J. Cogn. Enhanc. 2018, 2, 43-62. [CrossRef]

14. Wells, T.T.; Beevers, C.G. Biased Attention and Dysphoria: Manipulating Selective Attention Reduces Subsequent Depressive Symptoms. Cogn. Emot. 2010, 24, 719-728. [CrossRef]

15. Emerson, L.-M.; de Diaz, N.N.; Sherwood, A.; Waters, A.; Farrell, L. Mindfulness Interventions in Schools: Integrity and Feasibility of Implementation. Int. J. Behav. Dev. 2020, 44, 62-75. [CrossRef]

16. Carsley, D.; Khoury, B.; Heath, N.L. Effectiveness of Mindfulness Interventions for Mental Health in Schools: A Comprehensive Meta-Analysis. Mindfulness 2018, 9, 693-707. [CrossRef]

17. Mrazek, M.D.; Franklin, M.S.; Phillips, D.T.; Baird, B.; Schooler, J.W. Mindfulness Training Improves Working Memory Capacity and GRE Performance While Reducing Mind Wandering. Psychol. Sci. 2013, 24, 776-781. [CrossRef] [PubMed]

18. Mrazek, M.; Zedelius, C.M.; Gross, M.; Mrazek, A.I.; Phillips, D.T.; Schooler, J. Mindfulness in Education: Enhancing Academic Achievement and Student Well Being by Reducing Mind-Wandering. In Mindfulness in Social Psychology; Routledge: London, UK, 2017; pp. 139-152.

19. Mrazek, A.J.; Mrazek, M.D.; Cherolini, C.M.; Cloughesy, J.N.; Cynman, D.J.; Gougis, L.J.; Landry, A.P.; Reese, J.V.; Schooler, J.W. The Future of Mindfulness Training Is Digital, and the Future Is Now. Curr. Opin. Psychol. 2019, 28, 81-86. [CrossRef] [PubMed]

20. Antonson, C.; Thorsén, F.; Sundquist, J.; Sundquist, K. Upper Secondary School Students' Compliance with Two Internet-Based Self-Help Programmes: A Randomised Controlled Trial. Eur. Child. Adolesc. Psychiatry 2018, 27, 191-200. [CrossRef]

21. Mrazek, A.J.; Mrazek, M.D.; Reese, J.V.; Kirk, A.C.; Gougis, L.J.; Delegard, A.M.; Cynman, D.J.; Cherolini, C.M.; Carr, P.C.; Schooler, J.W. Mindfulness-Based Attention Training: Feasibility and Preliminary Outcomes of a Digital Course for High School Students. Educ. Sci. 2019, 9, 230. [CrossRef]

22. Pashler, H.; Wagenmakers, E.-J. Editors' Introduction to the Special Section on Replicability in Psychological Science: A Crisis of Confidence? Perspect. Psychol. Sci. 2012. [CrossRef]

23. Wingen, T.; Berkessel, J.B.; Englich, B. No Replication, No Trust? How Low Replicability Influences Trust in Psychology. Soc. Psychol. Personal. Sci. 2020, 11, 454-463. [CrossRef]

24. Gross, J.J.; John, O.P. Individual Differences in Two Emotion Regulation Processes: Implications for Affect, Relationships, and Well-Being. J. Personal. Soc. Psychol. 2003, 85, 348-362. [CrossRef]

25. Gullone, E.; Taffe, J. The Emotion Regulation Questionnaire for Children and Adolescents (ERQ-CA): A Psychometric Evaluation. Psychol. Assess. 2012, 24, 409-417. [CrossRef] [PubMed]

26. Job, V.; Bernecker, K.; Miketta, S.; Friese, M. Implicit Theories about Willpower Predict the Activation of a Rest Goal Following Self-Control Exertion. J. Personal. Soc. Psychol. 2015, 109, 694-706. [CrossRef] [PubMed]

27. Smallwood, J.; Schooler, J.W. The Restless Mind. Psychol. Bull. 2006, 132, 946-958. [CrossRef] [PubMed]

28. Ralph, B.C.W.; Smith, A.C.; Seli, P.; Smilek, D. Yearning for Distraction: Evidence for a Trade-off between Media Multitasking and Mind Wandering. Can. J. Exp. Psychol. 2020, 74, 56-72. [CrossRef]

29. Asendorpf, J.B.; van de Schoot, R.; Denissen, J.J.A.; Hutteman, R. Reducing Bias Due to Systematic Attrition in Longitudinal Studies: The Benefits of Multiple Imputation. Int. J. Behav. Dev. 2014, 38, 453-460. [CrossRef] 